\title{
Prontuário Eletrônico do Paciente na educação médica: percepções de docentes e preceptores
}

\author{
Electronic Health Record in medical education: perceptions of teachers and preceptors
}

\author{
Ana Maria Pereira Rangel ${ }^{1}$ (D) | anamprangel@gmail.com \\ Miriam Struchiner ${ }^{1}$ (D) miriamstru@gmail.com \\ Gil Fernando Salles'1 (D) gilsalles@hucff.ufrj.br
}

\begin{abstract}
RESUMO
Introdução: A implantação do Prontuário Eletrônico do Paciente (PEP) em unidades hospitalares de ensino tem proporcionado a integração do uso de tecnologia de informação em saúde (TIS) na educação médica e na prática clínica.

Objetivo: Este estudo analisou a percepção de professores e preceptores-médicos, de uma universidade pública, sobre a integração do uso do PEP nas atividades práticas curriculares.

Método: Trata-se de um estudo de abordagem qualitativa. A coleta de dados foi realizada por meio de entrevistas semiestruturadas com seis professores e quatro preceptores de um curso de Medicina. O estudo adotou como categorias de análise os domínios de competências e os resultados de aprendizagem com o uso do PEP, identificados e aprimorados por um estudo multicêntrico inglês: saúde digital, acesso e geração de dados, comunicação, trabalho multiprofissional e acompanhamento e monitoramento. Adotou-se ainda a categoria "questões pedagógicas" para estimular a reflexão dos sujeitos da pesquisa sobre suas práticas pedagógicas com o PEP. Para análise dos dados, utilizou-se análise temática de conteúdo.

Resultado: O estudo apontou que os professores e preceptores identificaram a necessidade de orientação formal para que os discentes utilizem TIS no seu desenvolvimento educacional e profissional, na preservação do sigilo e da confidencialidade das informações, e no atendimento ao paciente. Para os sujeitos da pesquisa, o uso de sistemas de suporte à decisão associados ao PEP contribui para o processo de ensino-aprendizagem, além de possibilitar maior visibilidade das informações dos demais profissionais de saúde e o acompanhamento da história clínica dos pacientes pelos discentes. O PEP é uma ferramenta assistencial que tem potencial para promover o uso de metodologias ativas, pois contextualiza o ensino, permite autonomia e autoria aos discentes e os instiga na busca por conhecimento.
\end{abstract}

Conclusão: A integração curricular de TIS tem sido apontada como um caminho para o desenvolvimento de competências e habilidades clínicas dos discentes, quando estiverem utilizando o PEP nas unidades de prática clínica.

Palavras-chave: Prontuário Eletrônico do Paciente; Educação Médica; Tecnologia de Informação em Saúde.

\begin{abstract}
Introduction: The implementation of the Electronic Health Record (EHR) in hospital teaching units has promoted the integration of Health Information Technology (HIT) into medical education and clinical practice.

Objective: The study analyzed teachers and preceptors' perceptions about the integration of EHR in curricular practices at a public university.

Method: A qualitative study, in which data was collected through semi-structured interviews with six medical professors and four preceptors. The study adopted as categories of analysis the domains of competences and learning results from the use of EHR, identified and improved in a British multicenter study (Digital Health, Data Access and Generation, Communication, Multiprofessional Work, Accompaniment and Monitoring). A new category entitled "Pedagogical Issues", was included to stimulate subjects' reflections on their pedagogical practices with the EHR. Thematic content analysis was used for data analysis.

Results: Teachers and preceptors agree that students need formal guidance on how to use HIT in their educational and professional development, and to preserve the secrecy and confidentiality of information during patient care using the EHR. For them, the use of decision support systems associated with EHR contributes to the teaching-learning process, in addition to allowing greater visibility of information from other health professionals, and facilitates student access to patients' clinical data. EHR is a support tool that has the potential to promote the use of active methodologies, to contextualize teaching, to provide student autonomy and authorship, and to instigate them in the search for knowledge.
\end{abstract}

Conclusion: HIT curricular integration has been pointed out as a way for students to develop clinical competences and skills, when using EHR in their clinical practice units.

Keywords: Electronic Health Record; Medical Education; Health Information Technology.

${ }^{1}$ Universidade Federal do Rio de Janeiro, Rio de Janeiro, Rio de Janeiro, Brasil.

Editora-chefe: Rosiane Viana Zuza Diniz.

Editor associado: Jorge Carvalho Guedes.

Recebido em 08/06/21; Aceito em 03/10/21.

Avaliado pelo processo de double blind review. 


\section{INTRODUÇÃO}

Nas últimas décadas, as Diretrizes Curriculares Nacionais (DCN) têm modificado a educação nas escolas médicas brasileiras. As DCN orientam para uma formação médica que atenda às necessidades do Sistema Único de Saúde (SUS), com maior foco no processo de ensino-aprendizagem, na diversidade dos cenários de prática, na incorporação de metodologias ativas, na atenção às competências clínicas, no desenvolvimento do raciocínio clínico e na formação para o trabalho integrado a outras profissões da área da saúde. A tendência é um ensino voltado para a aquisição de competências, centrado no aluno e baseado na resolução de problemas, sempre com o intuito de integrar teoria e prática ${ }^{1,2}$.

Por sua vez, o uso de tecnologia de informação em saúde (TIS) em unidades de saúde tem potencial para favorecer as atividades nas áreas assistenciais, educacionais e na gestão dos recursos da saúde. Em hospitais voltados para as atividades de ensino, a implantação do Prontuário Eletrônico do Paciente (PEP) tem proporcionado benefícios ao processo de ensino-aprendizagem, contudo também tem apresentado preocupações com restrições de acesso aos alunos, oferecendo riscos ao desenvolvimento do raciocínio clínico dos discentes ${ }^{3,4}$.

A Association of American Medical Colleges (AAMC) enfatiza a importância do desenvolvimento de aprendizagem em TIS na graduação e na pós-graduação, e definiu um conjunto de indicadores para orientar o desenvolvimento de habilidades e competências cognitivas e comunicacionais para uso do PEP. A formação em documentação clínica com a utilização do PEP pode ser associada às principais competências educacionais para aprimorar, avaliar e monitorar o progresso dos discentes no acompanhamento de seus pacientes ${ }^{5}$.

No Reino Unido, um grupo de trabalho com docentes das áreas de Medicina, Farmácia, Enfermagem, Obstetrícia e Informática em Saúde de 12 instituições elaborou diretrizes para integrar o PEP ao ensino e identificou seis domínios de competência e 32 resultados de aprendizagem necessários para a educação de estudantes no contexto do PEP: saúde digital, acesso aos dados, geração de dados, comunicação, trabalho multiprofissional e acompanhamento e monitoramento ${ }^{6}$.

"Saúde digital" refere-se à necessidade de os alunos compreenderem as tecnologias disponíveis e o impacto delas no ambiente clínico. Quando surgem inovações tecnológicas ou mudanças no ambiente de ensino, para nortear as práticas educacionais, as instituições precisam prover formação e orientação para o uso significativo das TIS ${ }^{3,5-7}$. Os domínios "acesso aos dados" e "geração de dados" permitem que os estudantes de áreas da saúde acessem e interpretem os dados dos pacientes para tomada de decisões clínicas de forma efetiva ${ }^{6,8}$. "Comunicação" diz respeito à necessidade de os alunos interagirem de forma efetiva tanto com os demais profissionais de saúde quanto com seus pacientes no ambiente digital ${ }^{6,9,10}$. "Trabalho multiprofissional" envolve o reconhecimento e respeito dos alunos pela identidade profissional, pelos diferentes saberes e papéis da equipe de saúde, além da tomada de decisão compartilhada com outros profissionais de saúde no contexto do PEP ${ }^{3,6}$. O domínio "acompanhamento e monitoramento" refere-se à necessidade de os alunos considerarem as questões éticas envolvidas na visualização e no compartilhamento de dados dos pacientes no PEP, de modo a contribuir para a segurança das informações de saúde $e^{6,11,12}$.

Considerando que o uso do PEP nas atividades práticas, realizadas por alunos e internos, em unidades hospitalares interfere no processo de ensino-aprendizagem, este trabalho analisou a percepção de professores e preceptores-médicos de uma universidade pública sobre a integração do PEP nas atividades práticas curriculares.

\section{MATERIAIS E MÉTODOS}

O estudo foi desenvolvido no contexto de um hospital universitário (HU) que utiliza um modelo de PEP, que vem se aprimorando, há cerca de 20 anos, nas áreas de internação e no atendimento de urgência. Acolhe estudantes de Medicina nas disciplinas práticas que fazem acompanhamento ativo dos pacientes atendidos na instituição. Assim, os alunos de Medicina são orientados a criar login de acesso ao PEP a partir do quarto período, quando passam a frequentar o ambiente hospitalar. Os discentes utilizam o PEP no levantamento da história clínica, familiar, social e da doença atual, e na realização do exame físico dos pacientes, atividades que são desenvolvidas com a orientação e supervisão de professores e preceptores-médicos nas unidades assistenciais do hospital.

Após a apresentação do projeto de pesquisa para os professores do Departamento de Clínica Médica da Faculdade de Medicina e para os preceptores do Serviço de Clínica Médica, os responsáveis pelas unidades indicaram professores e preceptores que estavam desenvolvendo atividades práticas com alunos e internos nos setores de internação ou emergência do hospital. Foram indicados 12 professores e oito preceptores. Após duas tentativas de contato via e-mail e um contato direto, somente quatro preceptores e seis professores responderam ao convite para participar da pesquisa.

Com o objetivo de obter uma visão abrangente do objeto pesquisado, optou-se por um estudo de abordagem qualitativa. A coleta dos dados se desenvolveu com base em um roteiro semiestruturado, aplicado por meio de entrevistas com os professores e preceptores. Na elaboração do roteiro, utilizaram-se os domínios de competências e os resultados de aprendizagem identificados e aprimorados em estudo multicêntrico realizado 
no Reino Unido ${ }^{6}$, tanto no que diz respeito às questões de estudo quanto no que concerne à análise das entrevistas. Durante a realização da primeira entrevista (piloto), os pesquisadores observaram a tendência do entrevistado em dar mais foco a questões assistenciais do que ao uso do PEP na aprendizagem dos alunos. Assim, para enfatizar o uso educacional do PEP no processo de ensino-aprendizagem e instigar a reflexão dos sujeitos da pesquisa sobre suas práticas pedagógicas, foi incluída uma nova categoria: questões pedagógicas. Desse modo, este estudo buscou identificar os significados e a importância atribuída por docentes e preceptores às seis categorias e às 19 subcategorias apresentadas no Quadro 1.

As entrevistas foram transcritas e submetidas à análise temática do conteúdo ${ }^{13}$. A partir da exploração e organização das falas dos sujeitos e da definição do corpus do estudo, a análise relacionou os resultados com as categorias de análise estabelecidas, o quadro teórico e os objetivos propostos pela pesquisa.

No estudo, os professores são tratados como PF1, PF2... PF6, e os preceptores, como PR1, PR2... PR4, para preservar o anonimato dos participantes. Esta pesquisa recebeu parecer favorável do Comitê de Ética e Pesquisa (CEP), sob o número 3.437.517, de 4 de julho de 2019.

\section{RESULTADOS E DISCUSSÃO}

Os sujeitos deste estudo são seis professores do Departamento de Clínica Médica da Faculdade de Medicina e quatro preceptores do Serviço de Clínica Médica do HU. Os docentes atuam em enfermarias de Clínica Médica, Neurologia, Nutrologia e Hematologia e em Unidade de Terapia Intensiva, todos os preceptores atuam em enfermarias da Clínica Médica. Os docentes possuem, em média, 21 anos de atividade profissional, e os preceptores têm 12 anos. Em relação ao tempo de uso do PEP na educação médica, os docentes têm em média 20 anos; e os preceptores, 16.

A categoria "saúde digital" identificou a necessidade de uma orientação formal para que os discentes utilizem sistemas de informação em saúde para seu desenvolvimento educacional e profissional. Os professores e preceptores relataram que os alunos possuem intimidade com o uso de tecnologia e demonstram facilidade em aprender os recursos básicos do PEP. Contudo, ressaltaram a importância de os discentes receberem orientação para uso de recursos avançados no PEP, como o acesso aos dados epidemiológicos, de modo a contribuir para desenvolvê-los na área de pesquisa científica e na gestão de recursos públicos de saúde.

Eu acho que valeria a pena uma orientação formal para a pesquisa; para os alunos de iniciação científica é tudo, porque tem muitos recursos e dados para pesquisa no prontuário eletrônico que servem muito para as pesquisas científicas. Eu estou aprendendo agora com alguns alunos e residentes, porque, às vezes, a gente não usa tudo que o PEP pode trazer para a pesquisa (PF5).

Quadro 1. Categorias e subcategorias utilizadas na análise.

\section{Categorias}

1. Saúde digital

\section{Subcategorias}

1.1 Orientação formal e curricular para acesso aos sistemas de informação em saúde.

1.2 Riscos e benefícios da digitalização dos prontuários.

1.3 A interação dos alunos com sistemas de suporte à decisão clínica.

2. Acesso e geração de dados 2.1 Acesso efetivo e interpretação de dados para a tomada de decisões clínicas.

2.2 Entradas de dados corretas e completas no PEP para segurança e qualidade do cuidado.

2.3 Respeito pelo consentimento do paciente, pela privacidade e pela confidencialidade ao acessar dados.
3. Comunicação
3.1 O PEP durante o atendimento médico pode desviar o foco do paciente.
3.2 Facilitadores podem interferir na eficácia e na qualidade da comunicação escrita.

4. Trabalho multiprofissional $\quad 4.1$ Utilização das informações dos demais profissionais de saúde no PEP.

4.2 Integração dos alunos com a equipe multiprofissional.

5. Acompanhamento e monitoramento

5.1 Dados dos alunos no PEP podem dar suporte ao monitoramento para melhoria da qualidade do ensino médico.

5.2 Questões éticas com o acompanhamento de antigos pacientes pelos alunos.

6. Questões pedagógicas
6.1 O PEP pode ser utilizado como uma ferramenta pedagógica.

6.2 Acesso aos dados do PEP tem contribuído para o desenvolvimento do raciocínio clínico.

6.3 Uso de dados para o desenvolvimento de trabalhos acadêmicos.

6.4 O PEP favorece o uso de metodologias ativas. 
A literatura aponta que os alunos de Medicina recebem, no ambiente prático, a maior parte de sua educação em TIS. Entretanto, o aumento de investimentos em TIS tem impulsionado algumas escolas médicas a incluir disciplinas como informática biomédica em seus currículos ${ }^{7}$. Isso proporciona aos estudantes a oportunidade de desenvolver habilidades e competências em tecnologia de informação, com ênfase na segurança do paciente, no trabalho em equipe multiprofissional e no atendimento baseado em evidências, antes de frequentarem os ambientes práticos da educação médica ${ }^{4,6,7}$. A AAMC tem recomendado o treinamento dos alunos em TIS, aspecto ainda ausente em grande parte dos currículos médicos tradicionais ${ }^{5}$.

Durante a realização das entrevistas, os docentes e preceptores identificaram alguns benefícios que a digitalização dos prontuários proporciona à assistência e ao ensino: acessibilidade, disponibilidade, legibilidade, rastreabilidade e informações mais organizadas foram as características mais citadas como benefícios para o processo de aprendizagem dos alunos.

A informação, ela fica mais acessível e organizada para o acesso do aluno. Para conseguir buscar as informações, é mais fácil organizar a informação, ter tudo no mesmo lugar. Para a pesquisa é excelente, você tem o dado na sua mão, seja para mostrar para o aluno, seja para desenvolver pesquisa, seja para dar feedback ao aluno [...] (PF1).

A disponibilidade imediata aos registros médicos antigos e a outros dados parece, à primeira vista, ser um benefício da utilização do PEP. Contudo, essa facilidade pode fazer com que o aluno pesquise intensivamente o paciente antes de realmente examiná-lo. Esse atalho ao raciocínio clínico não apenas pode privar o estudante de uma experiência educacional, mas também pode trazer imprecisões na interpretação desses dados ${ }^{3}$.

Os professores e preceptores apontaram os riscos que o uso do PEP pelos alunos pode trazer para o processo de ensino. Os docentes consideram o acesso irrestrito aos dados e o sigilo das informações acessadas pelos alunos como os principais riscos. Já os preceptores consideram que o maior risco é o uso de mecanismos facilitadores como copiar e colar as informações. Os preceptores ressaltaram a importância da elaboração de evoluções autorais pelos discentes e o perigo de repetir informações sem questionamentos. O professor PF3 concorda com os preceptores e argumenta que a elaboração da evolução é um processo mental importante, que deve ser desenvolvido pelo aluno sem utilizar cópias de evoluções anteriores.

O único risco que eu vejo é o uso indevido do copiar e colar parar fazer a evolução do paciente, que é um processo mental importante, no meu modo de ver, para que o aluno pare e pense o que está acontecendo com esse paciente, está acontecendo isso, está evoluindo dessa maneira, dentro dessa maneira está melhorando, está piorando, isto é um processo que requer treinamento, que se aprimora com treinamento [...] (PF3).

A preocupação dos docentes e preceptores com o uso de dispositivos de copiar e colar anotações pelos discentes, que pode prejudicar o desenvolvimento do raciocínio clínico destes, converge com outros estudos que apontaram aumento da quantidade de informações inconsistentes e desatualizadas, contribuindo para a diminuição da eficácia da comunicação entre as equipes de saúde e impacta na segurança do paciente, além de prejudicar o desenvolvimento do raciocínio clínico e de evoluções autorais, reduzindo o feedback do preceptor ${ }^{3,4}$.

Os entrevistados consideram que o adoção do Computerized Clinical Decision Support System (CDSS) associado ao PEP pode ser uma oportunidade para os alunos aprenderem com orientação, de modo a diminuir erros e prover maior segurança ao cuidado do paciente. O CDSS tem potencial para aprimorar o conhecimento de alunos e residentes, pois permite acesso fácil à literatura médica relevante e atualizada, e possibilita a formulação de planos terapêuticos e diagnósticos com mais precisão ${ }^{3,4,14}$. Porém, estudos ressaltam que a adesão às sugestões do CDSS não garante a reflexão necessária à aprendizagem. É preciso que o aluno compreenda as razões pelas quais essas diretrizes foram estabelecidas ${ }^{3,6}$.

A categoria "acesso e geração de dados" apontou que os alunos demonstram interesse pelo PEP, que ao iniciar sua aprendizagem prática buscam obter acesso, que possuem intimidade com o uso da tecnologia e aprendem rápido a buscar todas as informações dos pacientes no processo de desenvolvimento do seu raciocínio clínico e na tomada de decisão.

Os professores e preceptores consideram que os alunos são comprometidos com a inclusão de informações de forma correta e completa no PEP, que são zelosos e cuidadosos ao escreverem suas evoluções no prontuário.

[...] quando os alunos utilizam o PEP, eu acho que eles estão muito mais nervosos, que abrem o sistema, tem uma senha, aparece o nome deles, entende? Eles estão muito mais comprometidos, tanto é que existe um efeito, que eles não escrevem direto no PEP, eles fazem no bloco de notas, e muitos deles ficam esperando alguém ver as anotações antes de gravar no PEP (PF4).

No ambiente hospitalar, a interação entre os diferentes profissionais de saúde acontece de modo dinâmico, mediado muitas vezes pelo prontuário do paciente, já que as anotações e prescrições são utilizadas pelos demais profissionais na execução de suas tarefas e na tomada de decisão ${ }^{8,14}$. Assim, a 
atuação da preceptoria na orientação dos alunos é fundamental para a segurança do paciente e para a redução do uso de mecanismos facilitadores que possam impactar negativamente a qualidade da informação inserida pelos discentes ${ }^{3,4}$.

A maioria dos docentes acredita que os alunos entendem a importância do sigilo das informações acessadas no PEP e têm consciência de que podem ser rastreados, pois sabem que, por meio do login, é possível saber quem e quando acessou uma determinada informação. Entretanto, os preceptores discordam quando afirmam que os alunos não têm essa consciência e acreditam que é papel do preceptor orientá-los sobre confidencialidade, privacidade e sigilo das informações de saúde dos pacientes sob os seus cuidados.

Eu acho que não há essa clareza não, não há clareza que o prontuário pertence ao paciente, que se o paciente estando lúcido e orientado, o prontuário só pode ser visto por ele, o profissional que está cuidando do paciente, não há muito essa discussão. As pessoas fotografam, eu acho que os alunos não têm essa consciência (PR2).

A literatura aponta que a instituição de ensino deve fornecer aos discentes orientações quanto à importância do sigilo das informações acessadas no ambiente hospitalar ${ }^{11,12}$. Atualmente, com a facilidade de uso de dispositivos móveis e da comunicação instantânea entre as pessoas, é importante orientar os alunos sobre o perigo da divulgação de informações, imagens e vídeos sem o devido consentimento dos indivíduos envolvidos. As DCN do curso médico enfatizam que os estudantes devem comunicar-se com pacientes, familiares, comunidade e equipe de saúde com empatia e sensibilidade, preservando a confidencialidade, compreensão, autonomia e segurança da pessoa sob cuidado².

Em relação à categoria "comunicação", os professores e preceptores revelaram suas preocupações com o uso do PEP durante o atendimento médico, que pode desviar o foco do paciente, causar distrações e gerar impacto negativo no processo de ensino. Orientação e treinamento são indicados como forma de mitigar o risco de distração e fazer com que o PEP não se transforme em uma barreira, um terceiro elemento na relação médico-paciente. Os alunos devem aprender a desenvolver o atendimento na consulta clínica e utilizar os recursos tecnológicos disponíveis para benefício do tratamento de saúde do paciente.

Em tese sim, seja o prontuário eletrônico ou o próprio telefone, que toca, que manda mensagem, estamos sujeitos hoje a muitos distratores, então digamos que com trabalho de conscientização, de educação, acho que a gente consegue suprir essas barreiras, é um risco, mas eu acho que é um risco contornável (PR1).
Neste sentido, as instituições norte-americanas de ensino médico têm promovido orientações e treinamentos com o propósito de preparar seus alunos para o atendimento ambulatorial enquanto utilizam o PEP. Os alunos são orientados a manter o contato visual com o paciente durante manipulação dos dados no PEP, prestando atenção na fala do paciente e procurando mover o monitor do dispositivo compartilhando as informações, envolvendo o paciente na visualização dos dados contidos no PEP durante a consulta médica ${ }^{9,10}$.

Ao responderem questões sobre o "trabalho multiprofissional", os professores e preceptores informaram que o PEP facilita o acesso às informações da equipe multiprofissional, pois os dados estão dispostos no mesmo espaço das informações do corpo médico. E isso contribuiu para despertar o interesse dos discentes pelo conhecimento das diferentes áreas de saúde contido no PEP.

[...] com enfermagem talvez não, não muda muita coisa em termos práticos, mas dos outros demais profissionais de saúde, psicologia, fonoaudiologia e fisioterapia, a evolução deles ficava um pouco mais perdida. Hoje em dia tem a mesma padronização da evolução médica, todas as evoluções ficam mais fáceis de procurar [...] (PF2).

A padronização das evoluções dos diferentes profissionais de saúde no PEP pode contribuir para uma maior visibilidade das informações da equipe de saúde. Porém, o trabalho multiprofissional requer não somente que diferentes profissões da saúde compartilhem o mesmo espaço de trabalho, mas também que ocorram intervenções interprofissionais. Ainda são pouco frequentes a discussão de casos e a tomada de decisão em equipe multiprofissional ${ }^{15,16}$. O PEP pode contribuir para que discentes de Medicina visualizem e valorizem as informações dos demais profissionais da área de saúde. Contudo, é preciso que o preceptor oriente, estimule e explore as possibilidades, contribuindo com as DCN do curso médico que orientam para a formação interprofissional, com reflexão sobre a prática e sobre a troca de saberes com outros profissionais da área da saúde e outras áreas do conhecimento, estimulando o trabalho colaborativo e a qualidade da atenção à saúde².

Nas questões sobre "acompanhamento e monitoramento", as respostas apontaram que os dados inseridos pelos alunos no PEP podem proporcionar suporte ao monitoramento da aprendizagem prática. Alguns participantes da pesquisa acreditam que seria possível avaliar uma evolução inicial e outra no final do período para verificar se ocorreu crescimento nas habilidades de documentação no PEP pelos alunos: se os registros foram pertinentes, se retrataram o real estado de saúde do paciente e se conseguiram interpretar os 
dados corretamente para montar sua evolução clínica. Porém, como indicado a seguir, alertam que é preciso o trabalho de correção e orientação do preceptor.

[...] eu acompanho alunos do sexto período, você consegue pegar as evoluções iniciais e as evoluções do final do período e vê a diferença, é bem significativa, há uma curva de aprendizado, a qualidade da informação é melhor, é claro que a gente tem que fazer aquele trabalho de ficar sempre corrigindo, para que ocorra o aperfeiçoamento e não ocorra a incorporação de vícios (PR1).

Não existir um consenso entre os entrevistados sobre o uso das anotações dos discentes para monitorar a qualidade do aprendizado, a literatura aponta que o PEP pode facilitar a supervisão das atividades práticas executadas pelos discentes, identificando áreas em que eles apresentam deficiência com mais eficácia do que o prontuário de papel ${ }^{3,4}$.

O acompanhamento da história clínica de antigos pacientes pelos discentes no PEP, sem que estes pacientes tenham autorizado o acesso aos seus dados de saúde, é visto com preocupação ${ }^{11,12}$. Em nosso estudo, os professores e preceptores apontaram que os alunos acompanham os dados de pacientes motivados por questões afetivas e por interesse nos casos, e para verificar o desfecho, a evolução do diagnóstico e o tratamento proposto. Eles acreditam que essa atividade tem potencial para o desenvolvimento do aprendizado dos discentes.

Sempre, eles vêm falar, ao menos na reunião do sexto período, eles vêm e "Olha! Eu olhei aquele paciente que estava precisando operar", "O que quer dizer isso?". Então, contribui para o aprendizado, eles querem saber, estava com anemia por quê? Eles mesmos vão buscar no PEP, "Olha o ferro está baixo, ol ha!", chamam a atenção, então contribui muito (PR2).

A literatura ressalta que o rastreamento de antigos pacientes no PEP pelos discentes pode ter objetivos educacionais ou pura curiosidade. Entretanto, é importante que as questões éticas sejam respeitadas. Esses rastreamentos ocorrem sem orientação institucional, um aprendizado autodirecionado e com poucas preocupações éticas com as informações de saúde dos pacientes contidas no PEP ${ }^{11}$.

Quanto às "questões pedagógicas", os professores apontaram que costumam utilizar dados de pacientes contidos no PEP na elaboração de suas aulas. Alguns docentes visualizam o PEP como uma ferramenta assistencial que tem potencial para beneficiar o ensino, pois ele apoia o processo de ensinoaprendizagem, fornece informações e permite que os discentes desenvolvam seu raciocínio clínico por meio das evoluções diárias inseridas na plataforma digital.

Os registros eletrônicos de saúde contêm várias propriedades que os tornam desejáveis como material de ensino. Compreender como os alunos utilizam o PEP pode ajudar os educadores a melhor projetar as interações com eles e proporcionar a oportunidade de aprendizagem contextualizada. Contudo, no ambiente hospitalar é fundamental a atuação da preceptoria para identificar as necessidades individuais de aprendizagem, evitando que os discentes somente valorizem o uso de tecnologias e a opinião de especialistas em detrimento do contato diário com os pacientes na beira do leito ${ }^{17}$.

A facilidade de acesso às informações no PEP é percebida pelos participantes da pesquisa como uma forma de transposição dos conteúdos teóricos para o cenário da prática. Os dados existentes no PEP, quando acessados pelos alunos, podem contribuir para instigá-los na busca por conhecimento científico. Os entrevistados acreditam que os dados do PEP podem ser utilizados pelos alunos de iniciação científica no desenvolvimento de suas pesquisas, além de despertar o interesse para a interpretação de indicadores epidemiológicos e gestão pública em saúde.

Um grande diferencial do prontuário eletrônico para o seu antecessor, o prontuário em papel, segundo a opinião dos participantes deste estudo, é sem dúvida a possibilidade de visualizar imagens. A visualização e manipulação das imagens radiológicas contribuem para o ensino, pois enriquecem a aprendizagem com a discussão dos casos com os radiologistas e ajudam a fazer a correlação com o laudo do exame elaborado pelo especialista.

Enorme, gigantesco, esse potencial é o maior de todos. É um sonho, um prazer você sentar na beira do leito e ver a tomografia que foi feita dez minutos antes. Em termos assistenciais, é maravilhoso, mas, em termos educativos, é sensacional, o aluno ver na hora, o paciente está ali, acabou de examinar o paciente e ver a tomografia na mesma hora (PF3).

A possibilidade de os alunos visualizarem as imagens de exames radiológicos e complementares, por meio do PEP, pode contribuir para o desenvolvimento do seu raciocínio clínico de forma contextualizada. Entretanto, esse potencial será melhor explorado conforme a atuação da preceptoria. É preciso estimular os alunos a questionar e elaborar considerações a respeito dos casos apresentados. Dessa forma, estarão corresponsabilizando-se pela própria formação, aprendendo a aprender com autonomia, como parte do processo de ensino-aprendizagem preconizado nas DCN do curso médico².

A aprendizagem no ambiente clínico tem muitos pontos fortes para o desenvolvimento de metodologias ativas. É focado em problemas reais, contextualizado, onde os alunos são motivados a participar ativamente, desenvolvendo 
atitude e comportamento profissional diante dos pacientes e da equipe de saúde. A pesquisa apontou que, para os professores e preceptores, o PEP contribui para o uso de metodologias ativas oferecendo dados para a discussão dos casos clínicos, permitindo a participação ativa e instigando o aluno na construção do seu conhecimento, na transposição do conhecimento teórico para o prático.

\section{CONSIDERAÇÕES FINAIS}

As competências para o uso do PEP, adotadas neste estudo, em sua maioria, oriundas de um estudo multicêntrico inglês ${ }^{6}$, abrangem os principais aspectos encontrados na literatura e contribuíram para o desenvolvimento de uma abordagem ampliada sobre o uso do PEP no ensino médico. A inclusão da categoria "questões pedagógicas" em nosso estudo permitiu que docentes e preceptores expressassem suas perspectivas sobre suas práticas pedagógicas e refletissem sobre as possibilidades educacionais no cenário clínico com o uso do PEP pelos discentes.

Os resultados desta pesquisa apontam características do PEP, valorizadas por professores e preceptores, que podem favorecer o ensino, tais como a facilidade e rapidez para acessar as informações clínicas dos pacientes em acompanhamento, o uso de sistemas de suporte à decisão e o acesso às informações da equipe multiprofissionais. Contudo, também identificam aspectos que podem trazer impactos negativos para a educação e que devem ser mitigados por meio de treinamento e orientação com a atuação da preceptoria, como o uso de mecanismos facilitadores e a pouca preocupação ética dos discentes com sigilo das informações acessadas no PEP.

O uso do dispositivo de copiar e colar, na percepção dos professores e preceptores, tem impactado negativamente a educação dos discentes. Nosso estudo apontou prejuízo no desenvolvimento de evoluções autorais e no raciocínio clínico, gerando informações inconsistentes e desatualizadas, diminuído a eficácia da comunicação entre os membros da equipe de saúde, impactando na segurança do paciente e diminuindo a qualidade da aprendizagem dos discentes. Nossos achados corroboram com a literatura que orienta para a diminuição do uso desses dispositivos pelos discentes no $\mathrm{PEP}^{3,4}$.

A formação curricular em TIS tem sido apontada como o caminho para o desenvolvimento de competências e habilidades clínicas dos alunos, quando estiverem utilizando o PEP, nas unidades de prática clínica ${ }^{3,6}$. Capacitação e orientação para discentes, professores e preceptores podem contribuir para potencializar o PEP na formação profissional ${ }^{3,4}$.

Como limitações desta pesquisa, apontam-se o fato de não ter investigado a percepção dos discentes e o envolvimento de apenas uma instituição de ensino e seu modelo de PEP, que, além da função assistencial, possibilita o pleno uso educacional. No entanto, a metodologia e abordagem deste estudo, assim como os resultados obtidos, oferecem elementos para continuar, aprofundar e ampliar o escopo desta investigação em outras instituições de formação médica e com base em outros modelos de PEP, o que os pesquisadores consideram uma importante recomendação a partir deste estudo.

\section{CONTRIBUIÇÃO DOS AUTORES}

Ana Maria Pereira Rangel participou da concepção e do desenho do estudo, da coleta e interpretação dos dados, e da redação do artigo. Miriam Struchiner orientou a pesquisa e participou da concepção e interpretação dos dados, da redação e revisão do artigo. Gil Fernando Salles participou da redação e revisão do artigo.

\section{CONFLITO DE INTERESSES}

Declaramos não haver conflito de interesses.

\section{FINANCIAMENTO}

Declaramos não haver financiamento.

\section{REFERÊNCIAS}

1. Batista NA, Vilela RQB, Batista SHSS. Educação médica no Brasil. São Paulo: Cortez; 2015.

2. Brasil. Diretrizes Curriculares Nacionais do Curso de Graduação em Medicina. Brasília: Ministério da Educação; 2014.

3. Schenarts PJ, Schenarts K. Educational impacto of the Electronic Medical Record. J Surg Educ. 2012;69(1):105-12.

4. Tierney MJ, Pageler NM, Kahana M, Pantaleoni JL, Longhurst CA. Medica education in the Electronic Medical Record (EMR) era: benefits, challenges, and future directions. Acad Med. 2013;88(6):748-52.

5. Stephens MB, Gimbel RW, Pangaro L. The RIME/EMR Scheme: an educational approach to clinical documentation in Electronic Medical Records. Acad Med. 2011;86(1):11-4.

6. Pontefract SK, Wilson K. Using electronic patient records: defining learning outcomes for undergraduate education. BMC Med Educ. 2019;19-30.

7. Triola MM, Freidman E, Cimino C, Geyer EM, Wiederhorn J, Mainiero C. Health information technology and the medical school curriculum. Am J Manag Care. 2010;16(1):54-6.

8. Hammoud MM, Margo K, Dalymple JL, Stewart RA, Ali II, Briscoe GW et al. Medical student documentation in Electronic Health Records: a collaborative statement from the alliance for clinical education. Teach Learn Med. 2012;24(3):257-66.

9. Silverman $\mathrm{H}, \mathrm{Ho}$ Y, Kaib S, Ellis WD, Moffitt MP, Chen Qi, et al. A novel approach to supporting relationship-centered care through Eletronic Health Record ergonomic traing in pre-clerkship medical education. Acad Med. 2014;89(9):1230-4.

10. Lee WW, Alkureishi ML, Wroblewski KE, Farnan JM, Arora VM. Incorporating the human touch: piloting a curriculum for patient-centered Electronic Health Record use. Med Educ Online. 2017;22(1):1396171.

11. Asgarova $S$, Mackenzie $M$, Bates J. Learning from patients: why continuity matters. Acad Med. 2017;92(11):S55-S60.

12. Brisson GE, Tyler PD. Medical student use of Eletronic Health Records to track former patients. JAMA Intern Med. 2016;176(9):1395-7. 
13. Bardin L. Análise de conteúdo. Lisboa: Edições 70; 2004.

14. Keenan CR, Nguyen HH, Srinivasan M. Electronic Medical Records and their impact on resident and medical student education. Acad Psychiatry. 2006;30(6):522-7.

15. Feuerwerker LCM. As identidades do preceptor: assistência, ensino, orientação. In: Ribeiro VMB, organizadora. Formação pedagógica de preceptores do ensino em saúde. Juiz de Fora: Editota UFJF; 2011, p. 37-46.
16. Batista NA. Educação interprofissional em saúde: concepções e práticas. Cad FNEPAS. 2012;2:25-8.

17. Chi J, Bentley J, Kugler J, Che JH. How are medical students using the Electronic Health Record (EHR)?: an analysis of EHR use on an inpatient medicine rotation. PLoS One. 2019;14(8):1-6. 